\title{
A rare case of rectal cancer with perianal metastasis: a case report
}

\author{
Takuto Ikeda ${ }^{1 *} \mathbb{D}$, Atsushi Nanashima ${ }^{2}$, Akiko Ichihara', Eiji Kitamura', Kenzo Nagatomo ${ }^{1}$ and Hiroyuki Tanaka ${ }^{3}$
}

\begin{abstract}
Background: Cancer metastasis from colon cancer to an anal fistula is very rare. We herein reported a rare case in which local excision was performed for metastatic anal fistula cancer originating from rectal cancer.

Case presentation: A 68-year-old man was referred to our institution with a diagnosis of rectal cancer. He had complained of anal fistula for 5 years. Based on a recent history of cerebral infarction, Hartmann's operation was performed to treat the rectal cancer after the administration of preoperative chemotherapy for 3 months. However, 1 month after Hartmann's operation, the anal fistula was found to have worsened. Pelvic magnetic resonance imaging (MRI) revealed tumor formation at the perianal lesion. Metastatic anal fistula cancer originating from the rectal cancer was diagnosed based on the examination of the biopsied tissue. We selected local excision because the anal tumor had not invaded the surrounding tissue. There has been no recurrence in the 31 months after the curative operation.

Conclusion: Metastatic cancer should be ruled out when treating left-sided colon cancer with anal fistula. Local excision is one possible treatment for metastatic anal fistula cancer.
\end{abstract}

Keywords: Metastatic anal tumor, Anal fistula, Colon cancer

\section{Background}

One of the reasons for local recurrence at the anastomotic site after colectomy is thought to be due to the implantation of exfoliated cancer cells in the raw mucosa during anastomosis. It is thought that intraluminal exfoliated cancer cell implantation into the normal colorectal mucosa never happens without a mucosal defect. However, cancer cell implantation may be induced in an anal fistula due to mucosal destruction resulting from chronic inflammation. Rare cases of metastatic anal fistula cancer from colorectal cancer have been reported in the literature, some of which were treated by abdomino-perineal resection [1-11]. We herein present a very rare case of locally resected metastatic anal fistula cancer originating from rectal cancer, which showed a good prognosis.

\section{Case presentation}

A 68-year-old man was referred to our institution with a diagnosis of rectosigmoid colon cancer. Colonoscopy

\footnotetext{
* Correspondence: hiro0806@med.miyazaki-u.ac.jp

${ }^{1}$ Division of Gastrointestinal, Endocrine and Pediatric Surgery, Department of Surgery, Faculty of Medicine, University of Miyazaki, 5200 Kihara,

Kiyotake-cho, Miyazaki City, Miyazaki 889-1692, Japan

Full list of author information is available at the end of the article
}

revealed a type 2 tumor at the rectosigmoid colon (Fig. 1a). Right hemiparesis was present as a result of two cerebral infarctions that had occurred during the 2 months prior to his first admission. After consultation with a neurologist, we considered that it was necessary to wait several months before performing a major operation due to the risk of inducing another cerebral infarction. However, advanced cancer would be expected to grow during the waiting period. We therefore considered that it was necessary to administer systemic chemotherapy to suppress tumor growth. We initially administered five courses of preoperative chemotherapy (mFOLFOX6). No cerebral events or severe adverse events occurred during the chemotherapy. Stable disease, according to the RESIST criteria, was achieved with the preoperative chemotherapy. A laparoscopic Hartmann's operation with D3 lymph node dissection was performed at 4 weeks after the last course of chemotherapy. The patient's postoperative course was good without any complications. The final diagnosis of the rectal cancer was Dukes B with well-differentiated tubular adenocarcinoma and rectal tumor invasion to the subserosa (T3) without lymphatic or venous invasion (Fig. 1b). The resected specimen was free from cancer cells. The patient had complained of a perianal abscess for 5 years 

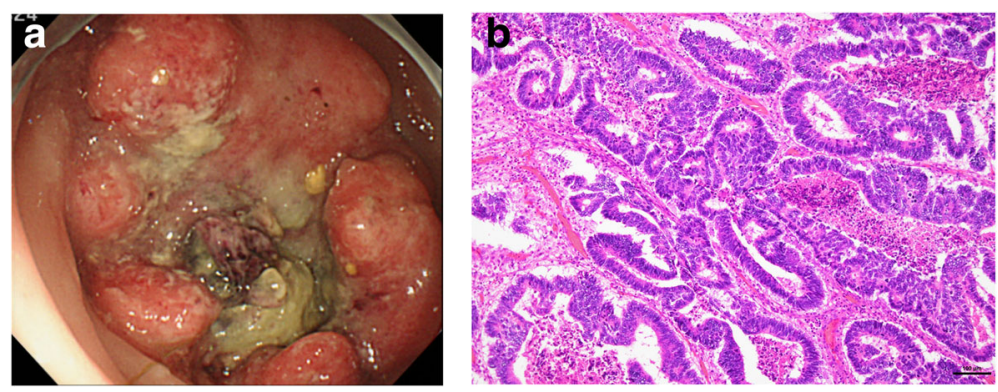

Fig. 1 a Colonoscopy revealed a type 2 tumor at the recto-sigmoid colon. $\mathbf{b}$ The surgically resected rectal specimen showed columnar or polygonal cells with hyperchromatic nuclei proliferating in tubular and cribriform patterns. Well-differentiated tubular adenocarcinoma was diagnosed

before his first admission. However, we gave priority to treating the rectal cancer. Although his anal symptoms did not worsen during chemotherapy, at 1 month after the Hartmann's operation for rectal cancer, his perianal abscess worsened and induration with two secondary open lesions was detected on physical examination (Fig. 2a). The pathological examination of the biopsied perianal tissue revealed adenocarcinoma. Because the perianal tumor was localized without invasion of the neighboring tissue on magnetic resonance imaging findings (Fig. 2b), and considering his physical states, we performed local excision under spinal anesthesia for curative resection. The final pathological diagnosis of the resected specimen was metastatic adenocarcinoma to an anal fistula originating from rectal cancer, and the resected margin was free from cancer cells (Fig. 3a-c). Immunohistochemistry revealed that both the rectal tumor and anal tumor were cytokeratin 7 (CK7) - and cytokeratin 20 (CK20) + (Fig. 4). Based on the pathology of the primary tumor and the absence of lymphatic or venous invasion, metastasis was considered to have occurred due to the implantation of exfoliated cancer cells. Seven courses of postoperative adjuvant chemotherapy with the same regimen have been administered. No recurrence of the tumor has been seen for 31 months after the operation. If a locally recurrent tumor had been found near the rectum and anus without distant metastasis during follow-up, laparoscopic resection of the tumor with the residual rectum and anus would have been attempted after considering the patient's performance status.

\section{Discussion}

Metastasis from colorectal cancer to an anal fistula is very rare. The mechanism is thought to involve the adherence of free cancer cells to the tract of the anal fistula followed by tumor proliferation and invasive growth. Destruction of the intestinal mucosa may induce the adherence of cancer cells. Mekata et al. reported that damage to mucosal sites from obstructive colitis could induce the luminal implantation of cancer cells [12]. Hubens et al. revealed that mucosal damage caused by biopsy in the presence of viable colon cancer cells resulted in mucosal implantation and intraluminal growth in one out of 30 rats [13].

In 1954, Guiss reported a case of implantation of cancer cells within an anal fistula from sigmoid colon cancer [1]. Since then, 22 cases of metastatic anal fistula

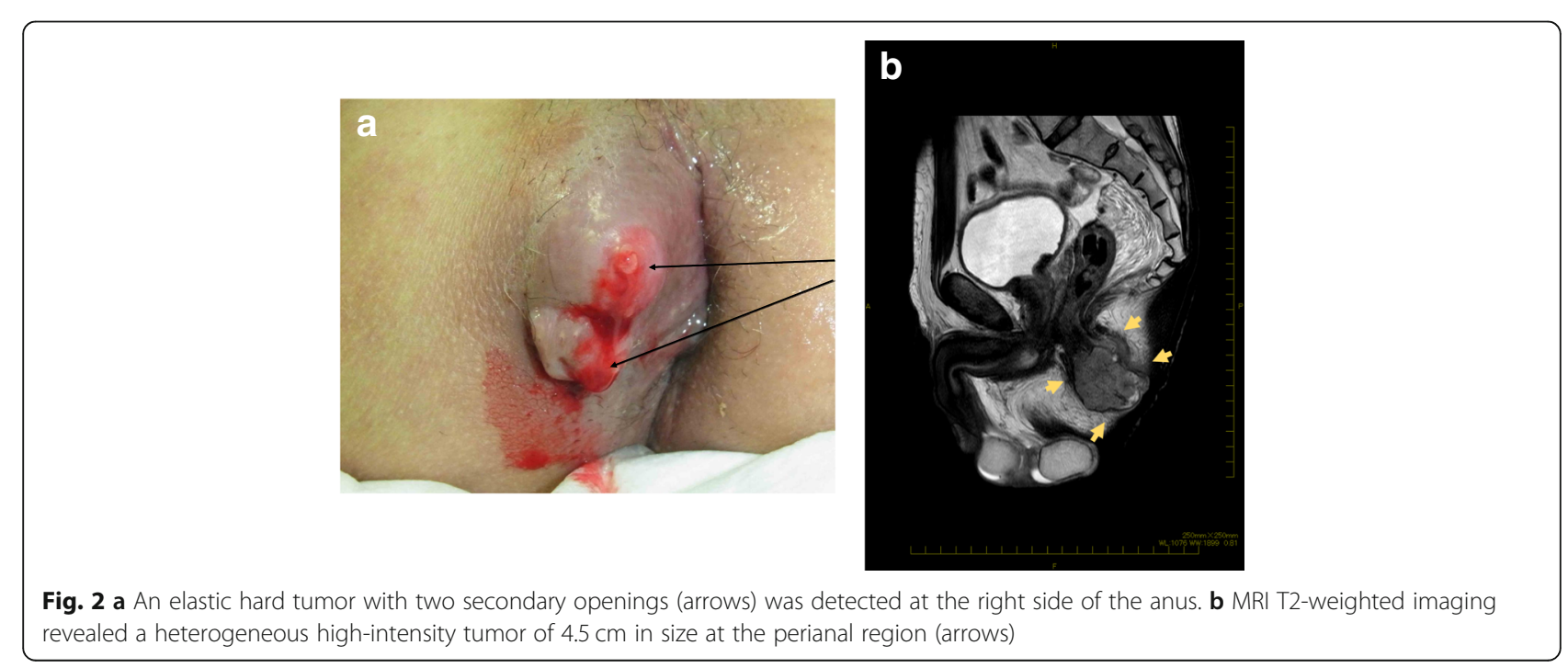



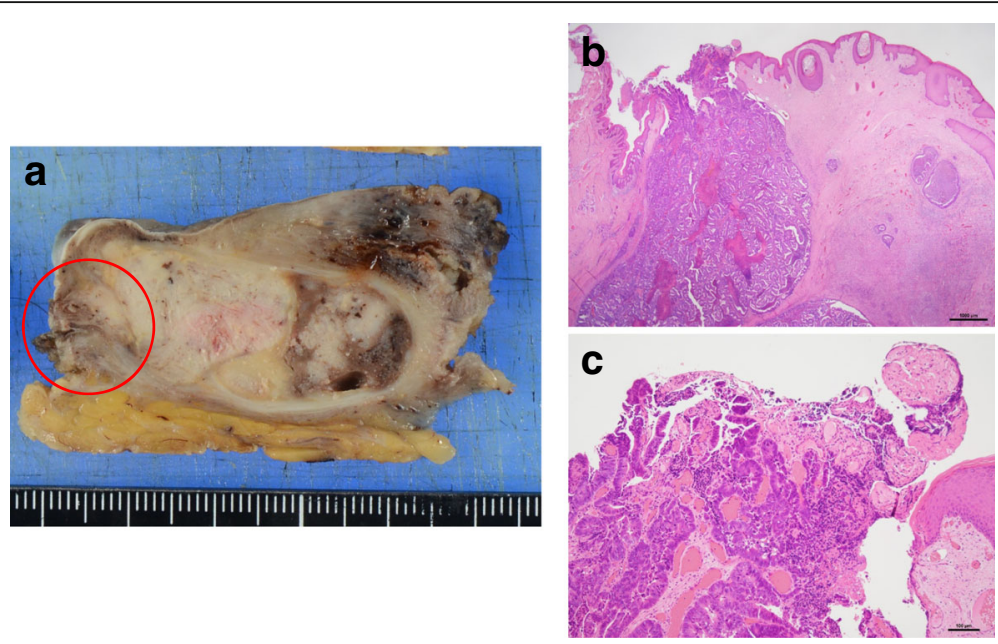

Fig. 3 a A resected specimen of the anal tumor. Macroscopically, the tumor grew in a capsulized cavity without invasive growth. The pathological findings of the encircled region in panel $\mathbf{a}$ are shown in panels $\mathbf{b}$ and $\mathbf{c}$. $\mathbf{b}$ Tumor growth without invasion to the surrounding tissue is detected. The apex of the tumor is exposed at the secondary opening $(\times 12.5)$. c Atypical epithelial cells with hyperchromatic nuclei and notable nuclei proliferate in a tubular pattern. Metastatic adenocarcinoma from rectal cancer was considered $(\times 100)$

cancer originating from colorectal cancer due to exfoliated cancer cell implantation have been reported in the English literature [2-11, 14-22]. We evaluated 24 cases, including our own (Table 1). All patients were males, and the tumors were located on the left-side colon, especially distal to the sigmoid colon. The period for which the anal fistula persisted before the diagnosis of the metastatic anal tumor ranged from 2 months to 20 years. Although most patients' anal tumors were pointed out synchronously with primary cancer, in four cases, they were detected at more than 1 year after surgery for the primary tumor.
When making a diagnosis of metastatic anal fistula, we must first rule out primary anal fistula cancer. Many cases were diagnosed based on the findings of hematoxylin-eosin staining. Moreover, as was used in our case, immunohistochemical staining of CK7 or CK20 has been used for differentiation in many cases $[7,8,10,11,17,18,21,22]$.

Radical resection of the primary tumor combined with the metastatic tumor has a crucial role in the treatment of metastatic anal fistula. Curative resection was performed in 21of 24 cases. Abdominoperineal resection (APR) was performed for 11 of 24 patients (45.8\%). On the other
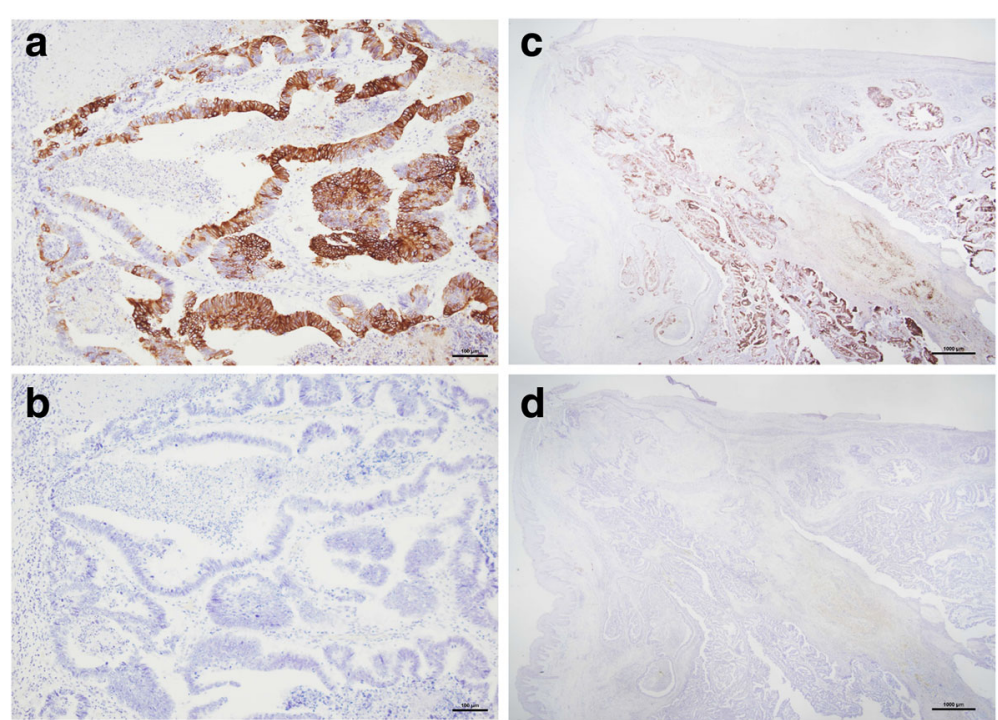

Fig.4 a Sections of the rectal tumor were immunopositive for cytokeratin $20(\times 100)$ and $\mathbf{b}$ immunonegative for cytokeratin $7(\times 100)$. Sections of anal fistula tumor were $\mathbf{c}$ immunopositive for cytokeratin $20(\times 12.5)$ and $\mathbf{d}$ immunonegative for cytokeratin $7(\times 12.5)$ 
Table 1 Evaluation of 24 cases

\begin{tabular}{|c|c|c|c|c|c|c|c|c|}
\hline & Gender & Age & Stage & Location & Operation & Sync or not & Chemo & Prognosis \\
\hline Guiss [1] & M & 63 & DukesA & $S$ & APR & Sync & None & 1 year, 2 months (-) \\
\hline Killingback et.al [2] & M & 47 & DukesA & $S$ & APR & NA & NA & NA \\
\hline Parnes [3] & M & 65 & DukesB & $S$ & APR & Sync & None & 18 months (-) \\
\hline Rollinson and Dundas [4] & M & 68 & NA & Rs & APR & Sync & None & 10 months (-) \\
\hline Thomas and Thompson [5] & M & 36 & DukesB & $\mathrm{S}$ & APR & Sync & NA & NA \\
\hline Isbister [14] & M & 39 & DukesC & $S$ & Hart+not rese & 12 months later & None & NA \\
\hline Isbister [14] & M & 47 & DukesC & Rs & AR+not rese & 12 months later & None & NA \\
\hline Isbister [14] & M & 69 & DukesC & Rs & By-pass & Sync & None & NA \\
\hline Shinohara et al. [15] & M & 66 & DukesC & $\mathrm{RaRb}$ & $L A R+L R$ & Sync & NA & 6 months liver meta \\
\hline Kouraklis et al. [6] & M & 47 & DukesB & $S$ & APR & Sync & NA & NA \\
\hline Hyman and Kida [7] & M & 44 & DukesB & $S$ & APR & Sync & None & 1 year (-) \\
\hline Zbar and Shenoy [16] & M & 72 & NA & S & $S+L R$ & Sync & CRT & 1 year, 2 months (-) \\
\hline Gupta et al. [17] & M & 53 & DukesC & Left colon & Lt. colec+LR & Sync & $5 F U / L V$ & 3 years $(-)$ \\
\hline Hamada et al. [18] & M & 53 & DukesB & $S$ & $A R+L R$ (coring out) & Sync & UFT/UZEL & 1 year $(-)$ \\
\hline Ishiyama et al. [19] & M & 53 & DukesC & $\mathrm{Ra}$ & $A R+L R$ & Sync & NA & 10 months died with carcin \\
\hline Sandiford et al. [20] & M & 72 & DukesB & Rs & $S+L R$ & Sync & CRT & 14 months (-) \\
\hline Gravante et al. [8] & M & 64 & DukesA & Left colon & Lt. colec, APR & 13 months later & CRT & 1 year $(-)$ \\
\hline Wakatsuki et al. [21] & M & 57 & DukesB & Rs & $A R+L R$ & 15 months later & None & 3 years, 7 months (-) \\
\hline Takahashi et al. [1 1] & M & 61 & NA & S & APR & Sync & 5FU/LV/CPT11 & 36 months $(-)$ \\
\hline Benjelloun et al. [22] & M & 68 & DukesB & Rs & $A R+L R$ & Sync & $\mathrm{NACRT} \rightarrow \mathrm{CR}$ & 3 years $(-)$ \\
\hline Benjelloun et al. [22] & M & 55 & DukesB & Rs & $A R+L R$ & Sync & $\mathrm{NACRT} \rightarrow \mathrm{CR}$ & 3 years $(-)$ \\
\hline Gomes et al. [10] & M & 65 & DukesB & $S$ & APR & Sync & None & 3 months $(-)$ \\
\hline Takahashi et al. [11] & M & 80 & DukesC & Ra & Lap APR & Sync & FOLFOX6 & 14 months (-) \\
\hline Our case & M & 68 & DukesB & Rs & Hart+LR & Sync & FOLFOX6 & 28 months (-) \\
\hline
\end{tabular}

$A P R$ abdomino-perineal resection, Hart Hartmann, not resect not resected, $A R$ anterior resection, $L R$ local resection, CRT chemoradiation, NACRT neoadjuvant chemoradiotherapy, Lt. colec left colectomy, Lap laparoscopic, sync synchronous, $S$ sigmoid colon, $R$ s recto-sigmoid colon, $R a$ upper rectum, $R b$ lower rectum, $N A$ not available

hand, including our case, 10 patients $(41.6 \%)$ were treated with local resection of the anal tumor as an anuspreserving curative operation [15-22]. Among 10 patients, 7 patients underwent perioperative chemotherapy or chemoradiotherapy. All of these patients showed a good prognosis without recurrence. Local resection can be selected for a localized anal fistula tumor with no invasive growth.

There is some debate as to whether surgery should be performed for primary cancer and metastatic anal cancer at the same time or at a different time. We are of the opinion that surgery for the primary rectal cancer should be performed first. If we had operated on the anal fistula first, the anal wound could have induced local recurrence due to the migration of exfoliated cancer cells from the primary rectal cancer. Simultaneous procedures might induce local recurrence at the perianal surgical wound. Moreover, patients with anal tumors must be carefully observed for re-recurrence for 1 to 2 years after a radical operation. During the operative procedure for the anal tumor, care must be taken to prevent the migration of cancer cells into the incision. During our patient's operation, we covered the anal tumor with gauze, which was sutured to the skin around the tumor.

The prognosis in these advanced cases is not so severe, with many of the reported patients surviving without recurrence. Perioperative adjuvant and neoadjuvant therapy, which is indicated in some cases, may improve the prognosis. Furthermore, a metastatic route that does not involve lymphovascular invasion may be another reason for the good prognosis. Knowledge of the initial symptom related to a perianal tumor may contribute to the early diagnosis of colon cancer. In many of the reported cases, the follow-up time was inadequate. Thus, the accumulation of additional data from cases with long-term follow-up is needed.

\section{Conclusion}

We presented a very rare case of a metastatic anal fistula tumor originating from colon cancer. Treatment for colorectal cancer in patients with an anal fistula must be 
performed in consideration of the possible development of a metastatic anal fistula tumor. Although an extended operation leaving no residual tumor is an important means of treatment, local resection should not be excluded for non-invasive tumors or in cases involving patients with severe complications.

\section{Acknowledgements}

Not applicable

\section{Authors' contributions}

$\mathrm{TI}$ and AN designed the report. HT performed the pathological analysis. EK and KN collected the patient's clinical data and information. TI and Al wrote the paper. All authors read and approved the final manuscript.

\section{Funding}

None

\section{Availability of data and materials}

All data generated or analyzed are included in this published article.

\section{Ethics approval and consent to participate}

Not applicable

\section{Consent for publication}

Consent for publication was obtained from the patient described in this article.

\section{Competing interests}

The authors declare that they have no competing interests.

\section{Author details}

${ }^{1}$ Division of Gastrointestinal, Endocrine and Pediatric Surgery, Department of Surgery, Faculty of Medicine, University of Miyazaki, 5200 Kihara, Kiyotake-cho, Miyazaki City, Miyazaki 889-1692, Japan. ${ }^{2}$ Division of Hepato-Biliary-Pancreas Surgery, Department of Surgery, Faculty of Medicine, University of Miyazaki, City, Miyazaki, Japan. ${ }^{3}$ Section of Oncopathology and Regenerative Biology, Department of Pathology, Faculty of Medicine,

University of Miyazaki, City, Miyazaki, Japan.

Received: 26 June 2019 Accepted: 12 August 2019

Published online: 20 August 2019

\section{References}

1. Guiss RL. The implantation of cancer cells within a fistula in ano: case report. Surgery. 1954:36:136-9.

2. Killingback M, Wilson $E$, Hughes ES. Anal metastases from carcinoma of the rectum and colon. Aust N Z J Surg. 1965;34:178-87.

3. Parnes $\mathbb{H}$. An interesting case of cancer of the sigmoid with concomitant cancer in an anal fistula. Mt Sinai J Med. 1976;43:476-9.

4. Rollinson PD, Dundas SA. Adenocarcinoma of sigmoid colon seeding into pre-existing fistula in ano. Br J Surg. 1984;71:664-5

5. Thomas DJ, Thompson MR. Implantation metastasis from adenocarcinoma of sigmoid colon into fistula in ano. J R Soc Med. 1992;85:361

6. Kouraklis G, Glinavou A, Kouvaraki M, Raftopoulos J, Karatzas G. Anal lesion resulting from implantation of viable tumour cells in a pre-existing anal fistula. A case report. Acta Chir Belg. 2002:102:212-3.

7. Hyman N, Kida M. Adenocarcinoma of the sigmoid colon seeding a chronic anal fistula: report of a case. Dis Colon Rectum. 2003;46:835-6.

8. Gravante G, Delogu D, Venditti D. Colosigmoid adenocarcinoma anastomotic recurrence seeding into a transsphincteric fistula-in-ano: a clinical report and literature review. Surg Laparosc Endosc Percutan Tech. 2008;18:407-8.

9. Takahashi H, Ikeda M, Takemasa I, Mizushima T, Yamamoto H, Sekimoto M, et al. Anal metastasis of colorectal carcinoma origin: implications for diagnosis and treatment strategy. Dis Colon Rectum. 2011:54:472-81.

10. Gomes RM, Kumar RK, Desouza A, Saklani A. Implantation metastasis from adenocarcinoma of the sigmoid colon into a perianal fistula: a case report. Ann Gastroenterol. 2014;27:276-9.
11. Takahashi R, Ichikawa R, Ito S, Mizukoshi K, Ishiyama S, Sgimoto K, et al. A case of metastatic carcinoma of anal fistula caused by implantation from rectal cancer. Surg Case Rep. 2015;1:123.

12. Mekata $E$, Shimizu $T$, Endo $Y$, Tani $T$. The rapid growth of intraluminal tumor metastases at the intestinal wall sites damaged by obstructive colitis due to sigmoid colon cancer: report of a case. Surg Today. 2008;38:862-5.

13. Hubens $G$, Lafullarde $T$, Van Marck E, Vermeulen P, Hubens A. Implantation of colon cancer cells on intact and damaged colon mucosa and serosa: an experimental study in the rat. Acta Chir Belg. 1994;94:258-62.

14. Isbister WH. Unusual 'recurrence' sites for colorectal cancer. Dig Surg. 2000;17:81-3.

15. Shinohara T, Hara H, Kato Y, Asano M, Nakazawa Y, Kato T, et al. Implantation of rectal cancer cells in a fistula in ano: report of a case. Surg Today. 2001;31:1094-6.

16. Zbar AP, Shenoy RK. Synchronous carcinoma of the sigmoid colon and a perianal fistula. Dis Colon Rectum. 2004:47:544-5 author reply 5.

17. Gupta R, Kay M, Birch DW. Implantation metastasis from adenocarcinoma of the colon into a fistula-in-ano: a case report. Can J Surg. 2005;48:162-3.

18. Hamada M, Ozaki K, Iwata J, Nishioka Y, Horimi T. A case of rectosigmoid cancer metastasizing to a fistula in ano. Jpn J Clin Oncol. 2005;35:676-9.

19. Ishiyama S, Inoue S, Kobayashi K, Sano Y, Kushida N, Yamazaki Y, et al. Implantation of rectal cancer in an anal fistula: report of a case. Surg Today. 2006;36:747-9.

20. Sandiford N, Prussia PR, Chiappa A, Zbar A. Synchronous mucinous adenocarcinoma of the rectosigmoid seeding onto a pre-existing anal fistula. Int Semin Surg Oncol. 2006:3:25.

21. Wakatsuki K, Oeda Y, Isono T, Yoshioka S, Nukui Y, Yamazaki K, et al. Adenocarcinoma of the rectosigmoid colon seeding into pre-existing anal fistula. Hepatogastroenterology. 2008;55:952-5.

22. Benjelloun el B, Aitalalim S, Chbani L, Mellouki I, Mazaz K, Aittaleb K. Rectosigmoid adenocarcinoma revealed by metastatic anal fistula. The visible part of the iceberg: a report of two cases with literature review. World J Surg Oncol. 2012;10:209.

\section{Publisher's Note}

Springer Nature remains neutral with regard to jurisdictional claims in published maps and institutional affiliations.
Ready to submit your research? Choose BMC and benefit from:

- fast, convenient online submission

- thorough peer review by experienced researchers in your field

- rapid publication on acceptance

- support for research data, including large and complex data types

- gold Open Access which fosters wider collaboration and increased citations

- maximum visibility for your research: over $100 \mathrm{M}$ website views per year

At $\mathrm{BMC}$, research is always in progress.

Learn more biomedcentral.com/submissions 